CLINICAL STUDY

\title{
Autonomic nervous system activity in diabetic and healthy obese female subjects and the effect of distinct weight loss strategies
}

\author{
Mirjam A Lips, Gerrit H de Groot ${ }^{1}$, Marieke De Kam ${ }^{2}$, Frits J Berends ${ }^{3}$, Renee Wiezer ${ }^{4}$, Bart A Van Wagensveld ${ }^{5}$,
} Dingeman J Swank ${ }^{6}$, Arijan Luijten ${ }^{7}$, Hanno Pijl and Jacobus Burggraaf ${ }^{2}$

Department of Endocrinology and Metabolism, Leiden University Medical Center, C-04-R-83, Albinusdreef 2, PO Box 9600,2333 ZA Leiden, The Netherlands, ${ }^{1}$ Department of Gastroenterology, Rode Kruis Ziekenhuis, Vondellaan 13, 1942 LE Beverwijk, The Netherlands, ${ }^{2}$ Centre for Human Drug Research, Zernikedreef 8, 2333 CL Leiden, The Netherlands, ${ }^{3}$ Department of Surgery, Rijnstate Ziekenhuis, Wagnerlaan 55, 6815 AD Arnhem, The Netherlands, ${ }^{4}$ Department of Surgery, St Antonius Ziekenhuis, PO Box 2500, 3430 EM Nieuwegein, The Netherlands, ${ }^{5}$ Department of Surgery, Sint Lucas Andreas Ziekenhuis Amsterdam, Jan Tooropstraat 164, 1061 AE Amsterdam, The Netherlands, ${ }^{6}$ Nederlandse Obesitaskliniek West, Harriët Freezerhof 183, 2492 JB Den Haag, The Netherlands and ${ }^{7}$ Department of Surgery, Maxima Medisch Centrum, De Run 4600, 5504 DB Veldhoven, The Netherlands

(Correspondence should be addressed to M A Lips; Email: m.a.lips@lumc.nl)

\begin{abstract}
Objective: Obesity and type 2 diabetes mellitus (T2DM) are reported to be associated with relative overactivity of the sympathetic nervous system (SNS), which is reversible by weight loss. However, direct effects of weight loss by calorie restriction vs Roux-en-Y gastric bypass (RYGB) on SNS overactivity were not studied in parallel. This study compared the effects of RYGB vs restrictive weight loss in obese patients with normal glucose tolerance (NGT) and with T2DM on SNS function as measured by heart rate variability (HRV).

Design and methods: Lean $(n=12)$, obese NGT $(n=27)$ and T2DM $(n=27)$ subjects were included in this study. Weight reduction in NGT subjects was achieved by gastric banding (GB) or RYGB and in T2DM subjects by RYGB or high-protein very-low-calorie diet (VLCD). HRV analysis was performed and blood samples were taken at baseline, 3 weeks and 3 months after intervention.

Results: At baseline, T2DM subjects showed SNS overactivity and NGT subjects showed similar, but non-significant, findings when compared with lean controls. Weight loss after 3 weeks was comparable in all treatment groups, whereas after 3 months, weight loss was most in VLCD and RYGB subjects. RYGB and VLCD treatment reduced SNS activity within 3 weeks in T2DM patients. After 3 months, restoration to normal autonomic nervous system activity was evident for all groups, except for the NGT-GB group.

Conclusion: We can conclude that SNS overactivity is more pronounced in obese T2DM subjects when compared with NGT subjects. Reduction of SNS overactivity coincides with weight loss with the timecourse of reduction dependent on the type of intervention. Surgery or caloric restriction may transiently induce SNS overactivity but do not prevent a direct restoration of sympathovagal balance.
\end{abstract}

European Journal of Endocrinology 169 383-390

\section{Introduction}

Both obesity and type 2 diabetes mellitus (T2DM) are marked by abnormal metabolic profiles (1). The pathogenesis of obesity and T2DM is incompletely understood but likely involves complex adaptations to chronic over-nutrition and inactivity (2). The autonomic nervous system (ANS) has a role in the regulation of long- and short-term energy balance, and ANS deregulation is implicated in the pathogenesis of obesity and T2DM (3). However, debate exists on the initiation of the pathogenic process: whether ANS deregulation is a pathogenic factor in the development of T2DM or whether, conversely, chronic hyperglycaemia and hyperinsulinaemia lead to ANS dysfunction.
Obesity and its early complications (i.e. insulin resistance and impaired fasting glucose) are associated with overstimulation of the sympathetic nervous system (SNS) and decreased tone of the parasympathetic nervous system (PNS) $(4,5)$. Long-term complications of T2DM are associated with chronic SNS overactivation (6). Furthermore, ANS dysfunction is also present in subjects preceding the onset of T2DM, suggesting that the development of ANS impairment parallels the development of obesity, hyperinsulinaemia and insulin resistance $(7,8,9)$ or has a pathogenic role in the development of diabetes $(10,11)$. Once T2DM has developed, chronic hyperglycaemia and persistent increase in sympathetic activity downregulate 
peripheral $\beta$-adrenergic receptors $(12,13)$, resulting in inability of the SNS to enhance energy expenditure (14).

Several trials showed that caloric restriction and weight loss have a beneficial influence on ANS dysfunction (15). However, to the best of our knowledge, no trials were performed to compare the effects of restrictive and 'metabolic' weight loss strategies in normal glucose tolerance (NGT) and T2DM obese subjects in parallel. Importantly, several factors including severe calorie restriction, surgery- and anaesthesia-induced stress, and hyperinsulinaemia after Roux-en-Y gastric bypass (RYGB) are suggested to have a stimulatory effect on the ANS (16).

ANS function can be assessed by heart rate variability (HRV) measurement, based on the interval variation between heart beats. Measures of HRV in both the time and frequency domain are reliable parameters to index the SNS-PNS balance (17).

The aim of this study was to assess the direct effect of surgery and caloric restriction and the effect of weight loss in groups of obese subjects with and without T2DM on ANS function as measured by HRV. We hypothesized that RYGB would have different sub-acute effects on ANS dysfunction when compared with calorie restriction per se. Furthermore, we hypothesized that recovery of ANS function would depend both on preoperative metabolic status and on the type of intervention.

\section{Subjects and methods}

\section{Subjects}

We included obese females eligible for both dietary and surgical treatment. Subjects eligible for surgical treatment were recruited from the waiting lists of several Dutch bariatric surgery centres. They had been screened previously by a multidisciplinary team of the Nederlandse Obesitaskliniek (Dutch Obesity Clinic) to establish whether they fulfilled the international criteria for bariatric surgery (18). Subjects eligible for dietary treatment were recruited after referral by their general practitioner or internist. They fulfilled the same criteria as surgical patients for BMI, co-morbidity, history of long-standing obesity and failed weight loss attempts. Exclusion criteria were smoking, age $>65$ years and any chronic disease other than diabetes, including psychiatric illness.

The subjects had either normal fasting glucose (NGT) or T2DM according to WHO standards. All diabetic subjects were treated with oral medication only (metformin or sulfonylurea derivatives). Subjects who reported the use of weight loss medications within 90 days prior to enrolment in the study were excluded. Body weight of all subjects had been stable for at least 3 months prior to inclusion in the study. Participants were allowed to use cholesterol-lowering statins and antihypertensive medication.
Control subjects were recruited via an advertisement. They were all healthy females, age matched to the obese subjects, with a BMI between 20 and $25 \mathrm{~kg} / \mathrm{m}^{2}$ and a normal plasma glucose concentration in fasting condition.

\section{Ethics}

The protocol was approved by the Medical Ethics Committee of the Leiden University Medical Center, and all subjects provided written informed consent before participation.

\section{Study design}

Initially, we intended to include obese NGT and T2DM subjects, who would have gastric banding (GB) or RYGB to systematically compare the physiological effects of these interventions. However, as RYGB was reported to have superior metabolic effects in subjects with T2DM, surgeons were reluctant to treat these subjects with GB (19). Instead, we chose to include a group of T2DM subjects who fulfilled the criteria for bariatric intervention and treated them with a very-low-calorie diet (VLCD). As the effects of GB presumably result primarily from calorie restriction, we reasoned that a VLCD might mimic the effects of GB.

Subjects were studied (after an overnight ( $10 \mathrm{~h}$ ) fast) within a month before surgery and again between 2 and 3 weeks after surgery. All oral glucose-lowering agents were discontinued for $48 \mathrm{~h}$. A cannula was inserted into an antecubital vein and a fasting blood sample was taken. Subjects were then given $266 \mathrm{ml}$ of a standardized fluid meal (Nutridrink, $400 \mathrm{kcal} ; 49$ energy\% carbohydrate $(48.9 \mathrm{~g}), 35 \%$ lipids $(15.4 \mathrm{~g})$ and $16 \%$ protein $(15.9 \mathrm{~g})$ ). Blood samples were drawn at the start of drinking $(t=0)$ and 5, 10, 20, 30, 60, 90, 120, 150 and 180 min postprandial. Blood was collected in a SST Gel and Clot Activator tube (Becton and Dickinson, Franklin Lakes, NJ, USA) and a vacutainer on EDTA. All blood samples, and serum samples when clotted, were centrifuged promptly $\left(2000 \boldsymbol{g}\right.$ at $4{ }^{\circ} \mathrm{C}$ for $\left.10 \mathrm{~min}\right)$ and subsequently plasma or serum was divided in separate plastic tubes and frozen $\left(-80^{\circ} \mathrm{C}\right)$ until assay.

\section{Surgery}

During RYGB, a $25 \mathrm{ml}$ gastric pouch was created and connected to a $100 \mathrm{~cm}$ Roux-en-Y limb. GB entailed placement of a standard silicone LapBand (Inamed, Allergan, Santa Barbara, CA, USA) around the stomach to create a $15 \mathrm{ml}$ pouch.

Patients increased their post-operative intake from a liquid diet for 5 days ( $<600 \mathrm{kcal} /$ day $)$ towards a more solid diet (700-800 kcal/day) after 3 weeks. Thereafter, patients followed the guidelines of The Dutch Obesity Clinic, ingesting up to $1200 \mathrm{kcal} /$ day. 


\section{Very-low-calorie diet}

Commercially available Prodimed (Prodimed Benelux BV, Valkenswaard, The Netherlands) is a high-protein low-calorie meal replacement plan (VLCD) with an average calorie intake of $600 \mathrm{kcal} /$ day. The meal replacement plan consists of sachets $(\sim 90 \mathrm{kcal}$ each of which contains $\sim 18 \mathrm{~g}$ protein, $\sim 2.5-5 \mathrm{~g}$ carbohydrates and $0.5-2 \mathrm{~g}$ fat) of soluble powder for preparation of meals. Subjects were allowed four to five sachets a day and an additional choice of selected vegetables $(600 \mathrm{kcal} /$ day in total) during the first 3 weeks. Up to 2 months, patients were allowed one piece of fruit and one portion of light dairy produce (800-1000 kcal/day in total). After 2 months, patients were allowed a light evening meal from prescribed recipes on intermittent days (1200 kcal/day in total).

\section{Use of medication}

On the day of operation or start of the VLCD, all blood glucose-lowering agents were discontinued to avoid hypoglycaemia. Only metformin treatment was reinstalled if fasting blood glucose levels remained above $7 \mathrm{mmol} / \mathrm{l}$ after intervention.

\section{Assays}

All samples were analysed after one freeze-thaw cycle. Serum glucose was measured using a Modular P800 chemistry analyzer of Roche Diagnostics with a coefficient of variation $(\mathrm{CV})$ of $1.7 \%$. Insulin was measured with an immunometric assay on an automated Immulite 2500 (Siemens, Breda, The Netherlands) with an intra-assay CV of $6-7.5 \%$. $\mathrm{HbA1c}$ was measured using an immunofluorometric assay on a Primus Ultra2 analyser with a CV of $0.5-$ $0.8 \%$. Leptin was measured using a RIA (Milipore, St Charles, MO, USA) with a CV of $3.4-4.7 \%$.

\section{Assessment of insulin resistance}

Insulin resistance was calculated with the homeostatic model assessment (HOMA2) (20) with the computer application downloaded from http://www.dtu.ox.ac.uk/ homacalculator/download.php.

\section{HRV measurement}

When patients had been in supine position for a minimum of $15 \mathrm{~min}$ and before or at least $30 \mathrm{~min}$ after blood sampling, a 5-min continuous recording 12-lead electrocardiogram (ECG) registration was made using the CardioPerfect ECG recording system (Cardiocontrol, Rijswijk, The Netherlands). Subjects were instructed to relax, to breathe regularly, not to speak and to stay awake. Each registration was screened for artefacts and subsequently analysed for HRV parameters according to the internationally accepted guidelines (17), using Kubios HRV Software vs 2 (Biosignal Analysis and Medical Imaging Group, Department of Physics, University of Kuopio, Kuopio, Finland). These parameters were in the time domain: mean $R$ wave to $R$ wave ( $R R$ ) interval; the square root of the mean squared difference of successive RR intervals (RMSSD; estimate of short-term components of HRV); the delta S.D.s of RR intervals, SD1 and SD2; and the ratio of SD1 over SD2 (balance of PNS/SNS tone) (17). The ECG recordings were also subjected to power spectrum analysis using the same software, but as these analyses showed the same outcome, these parameters are not further reported.

\section{Statistical analysis}

Data were analysed using SPSS 17.0. Data are presented as means \pm s.D. Differences between subject groups and lean controls at baseline were calculated using univariate ANOVA. The effects of the different interventions were compared with a mixed-effects model, with the patient groups and diabetes as fixed effects and the subject-specific deviances modelled with random intercepts. The Bonferroni's post hoc test was used to correct for multiple testing. A $P$ value $<0.05$ was considered statistically significant. Graphs were developed in Prism GraphPad 5 (La Jolla, CA, USA).

\section{Results \\ Subject characteristics}

All obese subjects and healthy controls were Caucasian females, with a mean age of $49.4 \pm 0.6$ years (Table 1 ). We included 32 subjects with T2DM and 30 NGT obese individuals. Eight subjects dropped out during the course of the study because they were not able to comply with the VLCD group $(n=2)$, because of practical issues $(n=3)$ and because of post-operative complications $(n=3)$.

Baseline characteristics of study groups are shown in Table 1. All parameters showed the expected differences between lean controls, obese NGT and obese T2DM subjects. Average time from diagnosis of T2DM at baseline was $3.7 \pm 2.5$ years in both T2DM groups.

\section{Weight loss and intake}

Average weight loss at 3 weeks was comparable after either intervention. After 3 months, RYGB and VLCD subjects had lost more weight $(\sim 15 \%)$ than NGT-GB subjects (Tables 2 and 3). According to protocol, patients expanded their intake after operation from a liquid diet for 5 days ( $<600 \mathrm{kcal} /$ day) towards a more solid diet (700-800 kcal/day) after 3 weeks. Thereafter, patients followed the intake guidelines of The Dutch 
Table 1 Baseline characteristics of study groups. Values are presented as mean \pm s.D. Differences between groups at baseline are calculated with univariate ANOVA. Least square difference (LSD) correction was applied to correct for multiple testing.

\begin{tabular}{|c|c|c|c|c|}
\hline & NGT (27) & DM (27) & Controls (11) & $P$ value \\
\hline Age (years) & $47.7 \pm 6.4$ & $51.0 \pm 7.1$ & $49.2 \pm 6.22$ & NS \\
\hline BMI $\left(\mathrm{kg} / \mathrm{m}^{2}\right)$ & $43.8 \pm 3.2$ & $42.0 \pm 5.5$ & $21.7 \pm 1.6$ & $<0.001$, T2DM/NGT vs controls \\
\hline Weight (kg) & $124.3 \pm 11.7$ & $117.2 \pm 17.1$ & $64.4 \pm 7.2$ & $<0.001$, T2DM/NGT vs controls \\
\hline $\mathrm{HbA} 1 \mathrm{c}(\mathrm{mmol} / \mathrm{mol})$ & $36.1 \pm 7.8$ & $49.6 \pm 12.0$ & $31.9 \pm 2.5$ & $<0.001$, T2DM vs controls/NGT \\
\hline HOMA-IR (\%) & $1.3 \pm 0.9$ & $1.6 \pm 1.1$ & $0.2 \pm 0.0$ & $<0.001, \mathrm{~T} 2 \mathrm{DM} / \mathrm{NGT}$ vs controls \\
\hline Fasting glucose $(\mathrm{mmol} / \mathrm{l})$ & $5.0 \pm 0.6$ & $8.7 \pm 2.5$ & $4.7 \pm 0.3$ & $<0.001$, T2DM vs controls/NGT \\
\hline Fasting insulin $(\mathrm{mU} / \mathrm{l})$ & $10.5 \pm 7.9$ & $12.0 \pm 7.8$ & $1.6 \pm 0.2$ & $<0.001$, T2DM/NGT vs controls \\
\hline Leptin $(\mu \mathrm{g} / \mathrm{l})$ & $86.0 \pm 39.8$ & $55.6 \pm 32.5$ & $9.9 \pm 5.0$ & $<0.001$, T2DM/NGT vs controls \\
\hline AỦC glucose $(\mathrm{mmol} / / / 3 \mathrm{~h})$ & $1037 \pm 167.8$ & $1843 \pm 546$ & $952 \pm 32.8$ & $<0.001$, T2DM vs controls/NGT \\
\hline AUC insulin (mU///3 h) & $7344 \pm 5741$ & $5821 \pm 2819$ & $2278 \pm 961$ & $<0.001, \mathrm{~T} 2 \mathrm{DM} / \mathrm{NGT}$ vs controls \\
\hline
\end{tabular}

Obesity Clinic, containing up to $1200 \mathrm{kcal}$ a day. Calorie intake in the diet group was similar to that in the surgically treated patients.

\section{Medication use}

All diabetic subjects discontinued their oral antidiabetics on the day of operation or start of the diet as per protocol. Metformin treatment was reinstalled in a similar percentage of patients in both diabetic groups (RYGB 27\% and VLCD 17\%). The use of other drugs such as statins and antihypertensive drugs was slightly higher in the diabetic subjects. At baseline, statins were used by $60 \%$ of T2DM patients and $25 \%$ of NGT patients. Of T2DM patients, $50 \%$ used antihypertensive drugs (diuretics, $n=7$; ACE inhibitors, $n=5$; and $\beta$-blockers, $n=6$ ) against $33 \%$ in NGT patients (diuretics, $n=4$; ACE-inhibitors, $n=3$; and $\beta$-blockers, $n=4$ ). Use of medication known to affect HRV parameters was nonsignificantly different in both groups and was continued throughout the entire study period.

To test the possible effect of concomitant medication with a possible influence on ANS tone, the statistical analyses were done separately for patients using or not using medication. These analyses showed similar results and therefore the data on the entire population are presented.

\section{Baseline characteristics of HRV parameters}

At baseline, T2DM subjects had a lower average RR interval compared with NGT subjects and lean controls (Table 4). RMSSD was decreased in T2DM subjects when compared with healthy controls $(P<0.05)$ and no significant decrease was observed when compared with NGT subjects $(P=0.053)$. SD1 and SD2 were lower in T2DM subjects compared with lean controls $(P=0.035$ and $P=0.005$ respectively) and, again, no significant decrease was observed when compared with NGT subjects $(P=0.053$ and $P=0.067)$.

\section{Effects of intervention within groups}

The effects of intervention within individual study groups are presented in Tables 2 and 3.

Table 2 Effects of intervention after 3 weeks and 3 months in NGT patients on anthropometrics, biochemical values and HRV parameters within and between study groups. Values are presented as mean \pm s.D. Differences within groups over time and in effect between the interventions were evaluated by mixed model analysis with individual 'baseline values' and 'T2DM/NGT' as covariates and patient groups (intervention) as fixed factor. Bonferroni's correction was used to correct for multiple testing.

\begin{tabular}{|c|c|c|c|c|c|c|}
\hline & \multicolumn{3}{|c|}{ NGT-GB $(n=11)$} & \multicolumn{3}{|c|}{ NGT-RYGB $(n=16)$} \\
\hline & Before & After 3 weeks & After 3 months & Before & After 3 weeks & After 3 months \\
\hline Weight (kg) & $118.6 \pm 12.9$ & $113.1 \pm 14.0^{\mathrm{a}}$ & $106.6 \pm 14.0^{\mathrm{a}}$ & $128.2 \pm 9.3$ & $119.4 \pm 10.2^{a}$ & $108.1 \pm 10.9^{a}$ \\
\hline $\mathrm{BMI}\left(\mathrm{kg} / \mathrm{m}^{2}\right)$ & $43.1+3.0$ & $40.5+2.9^{a}$ & $38.4+3.2^{\mathrm{a}}$ & $44.2+3.3$ & $40.9+3.6^{a}$ & $37.1+3.8^{\mathrm{a}}$ \\
\hline Weight loss (\%) & & $4.8 \pm 2.6$ & $10.2 \pm 3.4$ & & $6.9 \pm 3.0$ & $15.7 \pm 4.6^{\mathrm{b}}$ \\
\hline Glucose $(\mathrm{mmol} / \mathrm{l})$ & $5.0 \pm 0.6$ & $4.9 \pm 0.6$ & $5.2 \pm 0.8$ & $5.0 \pm 0.6$ & $4.8 \pm 0.8$ & $4.9 \pm 0.53$ \\
\hline Insulin (mU/I) & $11.5 \pm 8.7$ & $6.9 \pm 6.3$ & $10.0 \pm 7.2$ & $9.8 \pm 7.4$ & $8.8 \pm 9.3^{b}$ & $4.9 \pm 4.0^{\mathrm{a}}$ \\
\hline HOMA-IR (\%) & $1.5 \pm 1.1$ & $0.1 \pm 0.1^{a}$ & $0.2 \pm 0.1^{\mathrm{a}}$ & $1.3 \pm 0.9$ & $0.2 \pm 0.2^{\mathrm{a}}$ & $0.1 \pm 0.1^{a}$ \\
\hline Leptin $(\mu \mathrm{g} / \mathrm{l})$ & $70.6 \pm 25.4$ & $45.0 \pm 14.5$ & $42.6 \pm 15.5$ & $96.5 \pm 45.0$ & $55.9 \pm 30.7$ & $34.2 \pm 28.0$ \\
\hline Av. RR interval (ms) & $960.5 \pm 112.3$ & $970.3 \pm 110.0$ & $1014.3 \pm 155.5$ & $934.4 \pm 125.9$ & $963.3 \pm 139.6^{a}$ & $1058.0 \pm 170.9^{a}$ \\
\hline RMSSD (ms) & $42.7 \pm 20.6$ & $49.9 \pm 25.7$ & $44.8 \pm 23.0$ & $32.7 \pm 22.1$ & $37.9 \pm 25.3$ & $57.8 \pm 44.3^{\mathrm{a}}$ \\
\hline $\mathrm{SD} 1(\mathrm{~ms})$ & $30.3 \pm 14.6$ & $35.4 \pm 18.2$ & $31.7 \pm 16.6$ & $23.2 \pm 15.7$ & $26.8 \pm 17.9$ & $41.0 \pm 31.4^{\mathrm{a}}$ \\
\hline $\mathrm{SD} 2$ (ms) & $59.3 \pm 21.5$ & $59.3 \pm 17.2$ & $52.3 \pm 18.4$ & $51.5 \pm 20.9$ & $61.6 \pm 30.5$ & $68.6 \pm 23.9^{\mathrm{a}, \mathrm{b}}$ \\
\hline SD1/SD2 & $0.53 \pm 0.1$ & $0.59 \pm 0.2$ & $0.61 \pm 0.2$ & $0.42 \pm 0.2$ & $0.44 \pm 0.2$ & $0.58 \pm 0.4^{\mathrm{a}}$ \\
\hline
\end{tabular}

A $P$ value $<0.05$ was considered statistically significant.

a Significant effect of intervention within group (vs baseline).

${ }^{b}$ Significantly different effect between two interventions in either NGT or T2DM group. 
Table 3 Effects of intervention after 3 weeks and 3 months in DM patients on anthropometrics, biochemical values and HRV parameters within and between study groups. Values are presented as mean \pm s.D. Differences within groups over time and in effect between the interventions were evaluated by mixed model analysis with individual 'baseline values' and 'T2DM/NGT' as covariates and patient groups (intervention) as fixed factor. Bonferroni's correction was used to correct for multiple testing.

\begin{tabular}{|c|c|c|c|c|c|c|}
\hline & \multicolumn{3}{|c|}{ DM-RYGB $(n=15)$} & \multicolumn{3}{|c|}{ DM-VLCD $(n=12)$} \\
\hline & Before & After 3 weeks & After 3 months & Before & After 3 weeks & After 3 months \\
\hline Weight (kg) & $121.4 \pm 15.9$ & $112.5 \pm 15.1^{\mathrm{a}}$ & $100.9 \pm 13.4^{a}$ & $112.0 \pm 17.7^{\mathrm{a}}$ & $105.3 \pm 16.7^{a}$ & $97.2 \pm 16.1^{a}$ \\
\hline BMI $\left(\mathrm{kg} / \mathrm{m}^{2}\right)$ & $43.5 \pm 4.2$ & $40.4 \pm 4.0^{\mathrm{a}}$ & $36.1 \pm 3.8^{\mathrm{a}}$ & $40.2 \pm 6.4$ & $37.7 \pm 6.0^{\mathrm{a}}$ & $34.7 \pm 6.6^{\mathrm{a}}$ \\
\hline Weight loss (\%) & & $7.3 \pm 1.7$ & $16.9 \pm 2.1$ & & $6.0 \pm 1.5$ & $14.6 \pm 3.3$ \\
\hline Glucose $(\mathrm{mmol} / \mathrm{l})$ & $8.9 \pm 2.4$ & $6.7 \pm 1.5^{\mathrm{a}}$ & $5.8 \pm 1.2^{\mathrm{a}}$ & $8.4 \pm 2.6$ & $5.2 \pm 1.2^{\mathrm{a}}$ & $5.9 \pm 1.0^{\mathrm{a}}$ \\
\hline Insulin (mU/l) & $10.8 \pm 7.6$ & $10.9 \pm 6.2$ & $6.9 \pm 3.9^{\mathrm{a}}$ & $13.5 \pm 8.1$ & $4.9 \pm 2.9^{\mathrm{a}, \mathrm{b}}$ & $5.0 \pm 3.5^{\mathrm{a}}$ \\
\hline HOMA-IR (\%) & $1.6 \pm 1.1$ & $0.2 \pm 0.1^{\mathrm{a}}$ & $0.1 \pm 0.1^{\mathrm{a}}$ & $1.9 \pm 1.1$ & $0.1 \pm 0.1^{\mathrm{a}}$ & $0.1 \pm 0.1^{\mathrm{a}}$ \\
\hline Leptin $(\mu \mathrm{g} / \mathrm{l})$ & $65.7 \pm 38.6$ & $41.8 \pm 27.8$ & $33.7 \pm 39.8$ & $42.9 \pm 16.8$ & $22.0 \pm 14.5$ & $21.6 \pm 15.5$ \\
\hline Av. RR interval (ms) & $847.6 \pm 133.0$ & $943.3 \pm 137.7^{a}$ & $966.9 \pm 170.9$ & $837.2 \pm 123.0$ & $917.5 \pm 123.1^{\mathrm{a}}$ & $930.1 \pm 125.2^{\mathrm{a}}$ \\
\hline RMSSD (ms) & $27.7 \pm 15.9$ & $30.1 \pm 14.1$ & $36.0 \pm 15.7^{\mathrm{a}}$ & $24.4 \pm 11.3$ & $44.1 \pm 17.9^{\mathrm{a}, \mathrm{b}}$ & $39.0 \pm 16.3^{\mathrm{a}}$ \\
\hline $\mathrm{SD} 1$ (ms) & $19.6 \pm 11.2$ & $21.6 \pm 10.0$ & $25.5 \pm 11.2^{\mathrm{a}}$ & $17.3 \pm 8.0$ & $30.5 \pm 12.5^{\mathrm{a}, \mathrm{b}}$ & $31.3 \pm 15.6^{\mathrm{a}}$ \\
\hline SD2 (ms) & $48.6 \pm 28.5$ & $43.5 \pm 15.7$ & $44.3 \pm 19.0$ & $43.7 \pm 23.2$ & $57.9 \pm 27.1^{a, b}$ & $60.2 \pm 31.2^{\mathrm{a}, \mathrm{b}}$ \\
\hline SD1/SD2 & $0.44 \pm 0.2$ & $0.49 \pm 0.2$ & $0.60 \pm 0.2^{\mathrm{a}}$ & $0.40 \pm 0.1$ & $0.60 \pm 0.3^{\mathrm{a}}$ & $0.58 \pm 0.4^{\mathrm{a}}$ \\
\hline
\end{tabular}

A $P$ value $<0.05$ was considered statistically significant.

${ }^{\text {a }}$ Significant effect of intervention within group (vs baseline).

'Significantly different effect between two interventions in either NGT or T2DM group.

NGT subjects after GB No significant changes were induced by GB; however, SD1:SD2 ratio and the RR interval tended to increase after 3 months.

NGT subjects after RYGB In NGT subjects, RYGB increased all HRV parameters gradually, with differences reaching significance after 3 months.

DM subjects after RYGB In T2DM subjects, RR interval increased 3 weeks after RYGB. SD1, SD1/SD2 and RMSSD increased significantly after 3 months.

DM subjects after VLCD VLCD directly increased RR interval, RMSSD, SD1, SD2 and SD1/SD2. These effects remained significant at 3 months.

\section{Effects of intervention between groups}

RR interval was prolonged in T2DM subjects directly after RYGB and VLCD, whereas the effect of RYGB in NGT subjects was significant only after 3 months. RMSSD increased in T2DM subjects directly after VLCD, whereas after 3 months, RMSSD increased to the same extent in subjects following RYGB.

SD1, SD2 and SD1:SD2 ratio increased within 3 weeks in T2DM subjects after VLCD. After 3 months, SD1 increased to a comparable extent in RYGB subjects. In T2DM subjects, VLCD increased SD2 to a larger extent than RYGB. In NGT subjects, RYGB increased SD2 to a larger extent than GB. After 3 months, the effect on SD1/SD2 was comparable in RYBG and VLCD subjects but less in GB subjects.

\section{Correlations}

Glucose/insulin There was a correlation between fasting glucose and mean RR $(-0.48, P=0.000)$, $\operatorname{RMSSD}(0.442 ; P=0.001)$, SD1 $(0.441 ; P=0.001)$ and SD2 $(0.423 ; P=0.002)$ for all obese subjects. Area under the curve (AUC) glucose in the T2DM subjects also correlated with mean RR $(-0.441 ; P=0.021)$, $\operatorname{RMSSD}(0.570 ; P=0.002)$, SD1 $(0.570 ; P=0.002)$ and SD2 $(0.389 ; P=0.045)$.

\section{Discussion}

This is the first study to extensively evaluate the effect of RYGB compared with restrictive weight loss strategies on ANS function in matched groups of obese NGT and obese T2DM subjects. Subjects were studied within 3 weeks after surgery, when weight loss was limited, to compare the effect of the gastrointestinal rearrangement induced by RYGB to the effect of caloric restriction

Table 4 Baseline HRV parameters of study groups. Values are presented as mean \pm s.D. Differences between groups at baseline are calculated with univariate ANOVA. LSD correction was applied to correct for multiple testing.

\begin{tabular}{lcccc}
\hline & NGT $(n=27)$ & T2DM $(n=27)$ & Controls $(n=11)$ & $P$ value \\
\hline Av. RR interval $(\mathrm{ms})$ & $945.4 \pm 118.7$ & $842.8 \pm 126.3$ & $975.8 \pm 118.7$ & $<0.05$, DM vs NGT/controls \\
RMSSD (ms) & $37.0 \pm 21.7$ & $26.1 \pm 13.8$ & $37.6 \pm 13.9$ & $<0.05$, DM vs NGT/controls \\
SD1 (ms) & $26.2 \pm 15.4$ & $18.5 \pm 9.7$ & $26.6 \pm 9.9$ & $<0.05$, DM vs NGT/controls \\
SD2 (ms) & $54.8 \pm 21.1$ & $46.3 \pm 25.8$ & $67.9 \pm 22.6$ & $<0.05$, DM vs NGT/controls \\
SD1/SD2 & $0.47 \pm 0.2$ & $0.43 \pm 0.2$ & $0.40 \pm 0.1$ & NS \\
\hline
\end{tabular}


per se. Subjects were studied 3 months after surgery to evaluate the effect of marked weight loss. The principal finding of this study is in line with our hypothesis: SNS overactivity is more pronounced in obese subjects with T2DM when compared with obese subjects with a NGT. SNS overactivity decreases with weight loss, with the time-course of the effect depending on the type of intervention. Three months after intervention, this resulted in a comparable increase in PNS activity and restoration of the sympathovagal balance in subjects after RYGB or VLCD, whereas there was no effect in GB subjects.

ANS dysfunction in obesity and T2DM has generally been associated with sympathetic activation $(4,5,6)$, which, at least to some extent, is reversible by weight loss $(4,21,22)$. Two reciprocal hypotheses explain the possible pathophysiological mechanisms involved. In short, the hyperinsulinaemic or insulin-resistant state in obesity could cause ANS dysfunction, or conversely, pre-existing enhanced sympathetic tone causes hyperinsulinaemia and insulin resistance.

In insulin-sensitive patients, postprandial hyperinsulinaemia coincides with a transient SNS dominance enhancing resting energy expenditure (REE) to counteract hyperglycaemia $(23,24,25)$. At baseline, ANS function in our obese NGT subjects was not significantly different from lean controls, despite their hyperinsulinaemic but normoglycaemic state. Furthermore, SNS activity did not correlate with the enhanced insulin levels in the obese subjects. These observations do not support the notion that hyperinsulinaemia per se increases SNS activity. However, parameters of PNS activity correlated negatively with fasting and postprandial glucose in T2DM subjects. These observations suggest that insulin resistance alone may be related to unopposed overactivity of the SNS and that it is the subsequent rise in glucose levels in T2DM that impairs $\beta$-adrenergic signalling, reducing ANS responsiveness to variations in metabolic rate $(12,26)$. Taken together, and concordant with our baseline results, this suggests a gradual increase in SNS activation during evolution from obesity with a limited degree of hyperinsulinaemia, towards T2DM and development of overt SNS hyperactivity (9).

Conversely, some observations suggest a pathogenic role for SNS overactivity in the development and progression of insulin resistance $(10,13)$. SNS activation increases lipolysis, thereby enhancing free fatty acid release and inhibiting glucose transport. As well, adipose tissue-derived leptin causes sympathetic activation through a hypothalamic pathway that enhances REE (26). Selective leptin resistance in obesity (27), however, counteracts its metabolic effects, whereas the activating effect of leptin on the SNS is preserved (13, 28, 29). As hyperinsulinaemia, insulin resistance and hyperleptinaemia are hallmarks of obesity, it is difficult to define the initiator of this vicious circle. As we suggested, a combination of the two existent hypotheses may be true. Limited hyperinsulinaemia activates the SNS, and subsequent hyperglycaemia leads to neuronal damage to the unmyelinated fibres of the ANS $(30,31)$, whereas with progression of the disease also, myelinated nerve fibres will be damaged.

Our hypothesis that ANS dysfunction was reversible by weight loss, relative to the degree of weight loss and type of weight loss intervention, was confirmed by the results of our different treatment groups. Moreover, none of the HRV parameters of the study groups 3 weeks or months after intervention showed statistically significant differences with lean controls, suggesting a return to normal levels.

Subjects after RYGB showed a delay in PNS response when compared with those after VLCD. Indeed, intestinal rearrangements after RYGB enhance postprandial insulin release in the face of decreased postprandial glucose levels, suggesting a transient reduction in insulin sensitivity during the direct postoperative period that can be ascribed to transient increases in inflammation, tissue injury or perioperative starvation (32). In contrast, caloric restriction by VLCD or GB directly decreased fasting and postprandial glucose and insulin levels, probably by a reduction in endogenous glucose production (33). A comparable effect was achieved 3 months after RYGB, when insulin resistance was decreased following the loss in adipose tissue. From these observations, we suggest that a decline in insulin resistance per se is important for the recovery of autonomic function.

It has been suggested that calorie restriction or surgical stress has stimulating effects on the SNS (16). The overall increase in PNS activity in our subjects after VLCD and RYGB, however, does not support an important effect of caloric restriction or surgical stress. Furthermore, anaesthesia during surgery has profound suppressive effects on SNS or total ANS activity (34). It is unknown when these effects fade, as most studies only registered HRV during anaesthesia induction and recovery (34). We observed a gradual increase and decrease in PNS and SNS activity tone respectively in our RYGB patients even after 3 weeks. This contradicts a prolonged repressive effect of anaesthesia or suggests an effect that is only transient and does allow a continuous gradual increase in PNS over SNS activity. Also, we cannot rule out that mild post-operative complications such as pain and anxiety could have confounded the results in the surgical groups. However, although a small effect may have been present, as mentioned earlier, the gradual but persistent increase and decrease in PNS and SNS activity tone respectively in our RYGB subjects contradicts a strong effect of these complications.

Postprandial (but not fasting) GLP1 hypersecretion, enhancing glucose-stimulated insulin release, has been observed after RYGB and was present in our subjects as well (data not shown (35)). Rodent studies suggest a clear stimulating effect of GLP1 administration on SNS activity (36). Postprandial GLP1 hypersecretion after 
RYGB, but not after restrictive weight loss, may therefore induce a stimulatory effect on SNS activity postprandial after RYGB. The fact that we did not find correlations between GLP1 levels and HRV parameters, nor a stimulatory effect of RYGB on SNS activity in the fasting state, suggests that GLP1 did not affect our results. However, we cannot exclude an effect of GLP1 on postprandial HRV and this should be explored in future studies.

This study has some weaknesses. First of all, only female subjects were included, $80 \%$ of which were postmenopausal. This implicates that the results of this study apply only to female subjects. Moreover, we cannot rule out that differences in menstrual cycle (menopausal state) could have affected HRV measurements. However, we expected the a priori effect on HRV parameters which the interventions would cause to be much larger and as such to rule out possible influences due to menstrual cycle/menopausal state. Unfortunately, due to the large impact of GB and RYGB procedures, randomization of interventions was not allowed in our protocol. Moreover, two different interventions have been used in the calorie restriction group, which may have biased the effects one way or another. However, no clear evidence exists on metabolic effects of GB apart from those effects due to weight loss. Therefore, we suggest our comparison of these restrictive ways of weight loss against RYGB is valuable to at least some extent.

For obvious reasons, we were unable to restrict the study group to obese subjects not using any medication affecting autonomic regulation, such as $\beta$-blockers and ACE inhibitors, since these are frequently prescribed for concomitant hypertension in this study population. $\beta$-Blockers are known to affect heart rate and ANS tone, although the actual changes on HRV parameters are generally modest (37). Although we realize that we had a relatively small sample size, we performed our statistical analyses separately for patients taking or not taking medication. These analyses showed similar results and suggest that our findings are robust. We recognize, however, that we cannot completely rule out possible effects of the medication on our findings.

We conclude that SNS overactivity is more pronounced in obese T2DM subjects when compared with obese NGT subjects. This suggests a gradual increase in SNS activation caused by obesity, which may be associated with progressive insulin resistance and hyperinsulinaemia. This seems a dual process by hyperinsulinaemia, impairing $\beta$-adrenergic signalling and hyperglycaemia causing actual nerve fibre damage. Transient SNS overactivity may be induced by a surgically induced decrease in insulin sensitivity but does not prevent gradual and possibly lasting restoration of ANS tone after surgery. In general, reduction of SNS overactivity coincides with weight loss, with the time-course of reduction dependent on the type of intervention.

\section{Declaration of interest}

The authors declare that there is no conflict of interest that could be perceived as prejudicing the impartiality of the research reported.

\section{Funding}

This study was performed within the framework of the Center for Translational Molecular Medicine (http://www.ctmm.nl); project PREDICCt (grant 01C-104). The study was performed with an unrestricted grant from the Dutch Obesity Clinic.

\section{Author contribution statement}

M A Lips was involved in study concept, design, acquisition of data, analysis and interpretation of data, drafting of the manuscript and statistical analysis. G H de Groot was involved in study concept and design. M De Kam was involved in statistical analysis. F J Berends/ R Wiezer/B A Van Wagensveld/D J Swank/A Luijten were involved in acquisition of data. H Pijl was involved in study concept and design, analysis and interpretation of data. J Burggraaf was involved in study concept and design, analysis and interpretation of data and critical revision of the manuscript for important intellectual content.

\section{Acknowledgements}

The authors gratefully thank Elizabeth J M Ladan-Eijgenraam and Marjolein A Wijngaarden for their extensive clinical support while performing this study. The study could not have been performed without the efficient contribution of the clinical chemistry department (Bart E P B Ballieux and Jolanda C M Verhagen). They also thank Brigitte Bliemer, Sylvia Samsom, Puck Sickinger and Sandra Cosijns for facilitating patient inclusion.

\section{References}

1 Matthaei S, Stumvoll M, Kellerer M \& Haring HU. Pathophysiology and pharmacological treatment of insulin resistance. Endocrine Reviews 200021 585-618. (doi:10.1210/er.21.6.585)

2 Muoio DM \& Newgard CB. Mechanisms of disease: molecular and metabolic mechanisms of insulin resistance and $\beta$-cell failure in type 2 diabetes. Nature Reviews. Molecular Cell Biology 20089 193-205. (doi:10.1038/nrm2327)

3 Schwartz MW, Woods SC, Porte D Jr, Seeley RJ \& Baskin DG. Central nervous system control of food intake. Nature $2000 \mathbf{4 0 4}$ 661-671.

4 Emdin M, Gastaldelli A, Muscelli E, Macerata A, Natali A, Camastra S \& Ferrannini E. Hyperinsulinemia and autonomic nervous system dysfunction in obesity: effects of weight loss. Circulation 2001103 513-519. (doi:10.1161/01.CIR. 103.4.513)

5 Muscelli E, Emdin M, Natali A, Pratali L, Camastra S, Gastaldelli A, Baldi S, Carpeggiani C \& Ferrannini E. Autonomic and hemodynamic responses to insulin in lean and obese humans. Journal of Clinical Endocrinology and Metabolism $1998 \mathbf{8 3}$ 2084-2090. (doi:10.1210/jc.83.6.2084)

6 Cuspidi C, Meani S, Valerio C, Sala C, Fusi V, Zanchetti A \& Mancia G. Age and target organ damage in essential hypertension: role of the metabolic syndrome. American Journal of Hypertension 200720 296-303. (doi:10.1016/j.amjhyper.2006.09.010)

7 Festa A, D'Agostino R Jr, Hales CN, Mykkanen L \& Haffner SM. Heart rate in relation to insulin sensitivity and insulin secretion in nondiabetic subjects. Diabetes Care $2000 \quad 23$ 624-628. (doi:10.2337/diacare.23.5.624) 
8 Lehtinen JM, Uusitupa M, Siitonen O \& Pyorala K. Prevalence of neuropathy in newly diagnosed NIDDM and nondiabetic control subjects. Diabetes 198938 1307-1313. (doi:10.2337/diabetes. 38.10.1307)

9 Singh JP, Larson MG, O'Donnell CJ, Wilson PF, Tsuji H, Lloyd-Jones DM \& Levy D. Association of hyperglycemia with reduced heart rate variability (The Framingham Heart Study). American Journal of Cardiology 200086 309-312. (doi:10.1016/ S0002-9149(00)00920-6)

10 Mancia G, Bousquet P, Elghozi JL, Esler M, Grassi G, Julius S, Reid J \& Van Zwieten PA. The sympathetic nervous system and the metabolic syndrome. Journal of Hypertension 200725 909-920. (doi:10.1097/HJH.0b013e328048d004)

11 Straznicky NE, Eikelis N, Lambert EA \& Esler MD. Mediators of sympathetic activation in metabolic syndrome obesity. Current Hypertension Reports 200810 440-447. (doi:10.1007/s11906008-0083-1)

12 Bergholm R, Westerbacka J, Vehkavaara S, Seppala-Lindroos A, Goto T \& Yki-Jarvinen H. Insulin sensitivity regulates autonomic control of heart rate variation independent of body weight in normal subjects. Journal of Clinical Endocrinology and Metabolism 200186 1403-1409. (doi:10.1210/jc.86.3.1403)

13 Watanabe T, Nomura M, Nakayasu K, Kawano T, Ito S \& Nakaya Y. Relationships between thermic effect of food, insulin resistance and autonomic nervous activity. Journal of Medical Investigation 200653 153-158. (doi:10.2152/jmi.53.153)

14 Huggett RJ, Scott EM, Gilbey SG, Bannister J, Mackintosh AF \& Mary DA. Disparity of autonomic control in type 2 diabetes mellitus. Diabetologia 200548 172-179. (doi:10.1007/s00125004-1601-6)

15 Straznicky NE, Lambert GW \& Lambert EA. Neuroadrenergic dysfunction in obesity: an overview of the effects of weight loss. Current Opinion in Lipidology 201021 21-30. (doi:10.1097/MOL. Ob013e3283329c62)

16 Tentolouris N, Liatis S \& Katsilambros N. Sympathetic system activity in obesity and metabolic syndrome. Annals of the New York Academy of Sciences $2006 \mathbf{1 0 8 3} 129-152$. (doi:10.1196/annals. 1367.010)

17 Heart rate variability: standards of measurement, physiological interpretation and clinical use. Task Force of the European Society of Cardiology and the North American Society of Pacing and Electrophysiology. Circulation $1996 \quad 93 \quad 1043-1065$. (doi:10.1161/01.CIR.93.5.1043)

18 Fried M, Hainer V, Basdevant A, Buchwald H, Deitel M, Finer N, Greve JW, Horber F, Mathus-Vliegen E, Scopinaro N et al. Interdisciplinary European guidelines on surgery of severe obesity. International Journal of Obesity 200731 569-577.

19 Korner J, Bessler M, Inabnet W, Taveras C \& Holst JJ. Exaggerated glucagon-like peptide- 1 and blunted glucose-dependent insulinotropic peptide secretion are associated with Roux-en-Y gastric bypass but not adjustable gastric banding. Surgery for Obesity and Related Diseases 20073 597-601. (doi:10.1016/j.soard. 2007.08.004)

20 Levy JC, Matthews DR \& Hermans MP. Correct homeostasis model assessment (HOMA) evaluation uses the computer program. Diabetes Care 199821 2191-2192. (doi:10.2337/diacare.21. 12.2191)

21 Nault I, Nadreau E, Paquet C, Brassard P, Marceau P, Marceau S, Biron S, Hould F, Lebel S, Richard D et al. Impact of bariatric surgery - induced weight loss on heart rate variability. Metabolism 200756 1425-1430. (doi:10.1016/j.metabol.2007.06.006)

22 Perugini RA, Li Y, Rosenthal L, Gallagher-Dorval K, Kelly JJ \& Czerniach DR. Reduced heart rate variability correlates with insulin resistance but not with measures of obesity in population undergoing laparoscopic Roux-en-Y gastric bypass. Surgery for Obesity and Related Diseases 20106 237-241. (doi:10.1016/j. soard.2009.09.012)
23 Anderson EA, Hoffman RP, Balon TW, Sinkey CA \& Mark AL. Hyperinsulinemia produces both sympathetic neural activation and vasodilation in normal humans. Journal of Clinical Investigation 199187 2246-2252. (doi:10.1172/JCI115260)

24 Bellavere F, Cacciatori V, Moghetti P, Gemma ML, Dellera A, Tosi F, Negri C, Thomaseth K \& Muggeo M. Acute effect of insulin on autonomic regulation of the cardiovascular system: a study by heart rate spectral analysis. Diabetic Medicine 1996 13 709-714. (doi:10.1002/(SICI)1096-9136(199608)13:8<709::AIDDIA1 $58>3.0 . C O ; 2-8)$

25 Laitinen T, Vauhkonen IK, Niskanen LK, Hartikainen JE, Länsimies EA, Uusitupa MI \& Laakso M. Power spectral analysis of heart rate variability during hyperinsulinemia in nondiabetic offspring of type 2 diabetic patients: evidence for possible early autonomic dysfunction in insulin-resistant subjects. Diabetes 1999 48 1295-1299. (doi:10.2337/diabetes.48.6.1295)

26 Seals DR \& Bell C. Chronic sympathetic activation: consequence and cause of age-associated obesity? Diabetes 200453 276-284. (doi:10.2337/diabetes.53.2.276)

27 Correia ML, Haynes WG, Rahmouni K, Morgan DA, Sivitz WI \& Mark AL. The concept of selective leptin resistance: evidence from agouti yellow obese mice. Diabetes $2002 \quad 51 \quad 439-442$. (doi:10.2337/diabetes.51.2.439)

28 Rahmouni K, Haynes WG, Morgan DA \& Mark AL. Selective resistance to central neural administration of leptin in agouti obese mice. Hypertension 200239 486-490. (doi:10.1161/ hy0202.102836)

29 Rahmouni K. Leptin-induced sympathetic nerve activation: signaling mechanisms and cardiovascular consequences in obesity. Current Hypertension Reviews $2010 \quad 6 \quad 104-209$. (doi:10.2174/157340210791170994)

30 Brownlee M. Glycation products and the pathogenesis of diabetic complications. Diabetes Care 199215 1835-1843. (doi:10.2337/ diacare.15.12.1835)

31 Greene DA, Lattimer SA \& Sima AA. Are disturbances of sorbitol, phosphoinositide, and $\mathrm{Na}^{+}-\mathrm{K}^{+}$-ATPase regulation involved in pathogenesis of diabetic neuropathy? Diabetes $198837688-693$. (doi:10.2337/diabetes.37.6.688)

32 Thorell A, Nygren J \& Ljungqvist O. Insulin resistance: a marker of surgical stress. Current Opinion in Clinical Nutrition and Metabolic Care 19992 69-78. (doi:10.1097/00075197-19990100000012 )

33 Jazet IM, Pijl H, Frolich M, Romijn JA \& Meinders AE. Two days of a very low calorie diet reduces endogenous glucose production in obese type 2 diabetic patients despite the withdrawal of blood glucose-lowering therapies including insulin. Metabolism $2005 \mathbf{5 4}$ 705-712. (doi:10.1016/j.metabol.2004.12.015)

34 Ireland N, Meagher J, Sleigh JW \& Henderson JD. Heart rate variability in patients recovering from general anaesthesia. British Journal of Anaesthesia $1996 \mathbf{7 6}$ 657-662. (doi:10.1093/ bja/76.5.657)

35 Lips MA, de Groot GH, van Klinken J, Aarts E, Berends FJ, Janssen IM, Van Ramshorst B, Van Wagensveld BA, Swank DJ, Van Dielen F et al. Calorie restriction is a major determinant of the short-term metabolic effects of gastric bypass surgery in obese type 2 diabetic patients. Clinical Endocrinology 2013.

36 Yamamoto H, Lee CE, Marcus JN, Williams TD, Overton JM, Lopez ME, Hollenberg AN, Baggio L, Saper CB, Drucker DJ et al. Glucagon-like peptide-1 receptor stimulation increases blood pressure and heart rate and activates autonomic regulatory neurons. Journal of Clinical Investigation $200211043-52$.

37 Bilchick KC \& Berger RD. Heart rate variability. Journal of Cardiovascular Electrophysiology $2006 \quad 17 \quad 691-694$. (doi:10.1111/j.1540-8167.2006.00501.x)

Received 1 May 2013

Accepted 4 July 2013 